\title{
Connectivity dynamics of the Default Mode Network encode the stream of affect at multiple timescales
}

Giada Lettieri ${ }^{\mathrm{a}}$, Giacomo Handjaras ${ }^{\mathrm{a}}$, Francesca Setti ${ }^{\mathrm{b}}$, Elisa Morgana Cappello ${ }^{\mathrm{a}}$, Valentina Bruno ${ }^{\mathrm{c}}$, Matteo Diano $^{\mathrm{d}}$, Andrea Leo ${ }^{\mathrm{e}}$, Emiliano Ricciardi $^{\mathrm{b}}$, Pietro Pietrini ${ }^{\mathrm{b}}$ and Luca Cecchetti ${ }^{\mathrm{a}^{*}}$

a. Social and Affective Neuroscience Group, IMT School for Advanced Studies Lucca, Lucca, Italy.

b. Molecular Mind Laboratory, IMT School for Advanced Studies Lucca, Lucca, Italy.

c. MANIBUS Lab, Psychology Department, University of Turin, Turin, Italy.

d. Department of Psychology, University of Turin, Turin, Italy.

e. Department of Translational Research and Advanced Technologies in Medicine and Surgery, University of Pisa, Pisa, Italy.

* Corresponding Author:

Luca Cecchetti

IMT School for Advanced Studies Lucca

Piazza San Francesco, 19, 55100 Lucca - Italy

Email: luca.cecchetti@imtlucca.it 


\begin{abstract}
In everyday life, the stream of affect results from the interaction between past experiences, expectations and the unfolding of events. How the brain represents the relationship between time and affect has been hardly explored, as it requires modeling the complexity of everyday life in the laboratory setting. Movies condense into hours a multitude of emotional responses, synchronized across subjects and characterized by temporal dynamics alike real-world experiences.

Here, we use time-varying intersubject brain synchronization and real-time behavioral reports to test whether connectivity dynamics track changes in affect during movie watching. Results show that pleasantness and intensity of experiences relate to connectivity of the default mode network and converge in the right temporo-parietal cortex. We validate these results in two experiments including four independent samples, two movies and alternative analysis workflows. Lastly, we reveal chronotopic connectivity maps within temporo-parietal and fronto-polar cortex, where adjacent areas preferentially encode affect at specific timescales.
\end{abstract}


Emotions are intense and immediate reactions of the body and the brain to an external or internal event occurring in the present, happened in the past or that may occur in the future. In daily life, the temporal trajectory of emotions is characterized by regularities in features, such as the time to rise, the duration or the probability of resurgence (Kuppens and Verduyn, 2017), which inform mental models of emotion transitions and support affective forecasting (Thornton and Tamir, 2017).

Whence, it clearly appears that the study of diachronicity is crucial for building a more comprehensive understanding of emotion processing and conceptualization in humans. Indeed, over the last years, increasing attention has been dedicated to the study of the temporal characteristics of affect using behavioral and experience sampling methods (Trampe et al., 2015; Verduyn et al., 2015; Kuppens et al., 2010), which allow researchers to track emotions over days, weeks or months. In sharp contrast, studies on the brain correlates of affective dynamics are still limited (Waugh and Schirillo, 2012; Costa et al., 2014; Verduyn et al., 2015; Résibois et al., 2017; 2018) and have employed static or relatively brief stimuli (Posner et al., 2009; Baucom et al., 2012; Kim et al., 2017) that may not be adequate to account for the complex temporal trajectory of lifelike experiences (Waugh and Schirillo, 2012).

A possible strategy to overcome this limitation is measuring brain activity elicited by movies using functional magnetic resonance imaging (fMRI). Indeed, movies are ecological and dynamic stimuli that synchronize brain response across individuals (Hasson et al., 2004) and that are able to elicit a wide variety of emotional states in a few hours (Schaefer et al., 2010). In addition, movies represent a compelling tool to track time-varying brain connectivity (Nastase et al., 2019) and to reveal the interplay among large-scale networks. Promising evidence in this regard comes from a work that, using relatively brief movie excerpts ( $\sim 8$ minutes), demonstrated the association between connectivity of salience and amygdala-based networks and the perceived intensity of sadness, fear and anger (Raz et al., 2016).

Also, brain connectivity dynamics well exemplify the tight relationship between emotion and cognition, as the same brain region can interact with distinct functional networks over time and the 
bioRxiv preprint doi: https://doi.org/10.1101/2020.06.06.137851; this version posted March 1, 2021. The copyright holder for this preprint (which was not certified by peer review) is the author/funder, who has granted bioRxiv a license to display the preprint in perpetuity. It is made available under aCC-BY-ND 4.0 International license.

same network can support multiple mental processes (Pessoa, 2017; 2018). In line with this, studies combining naturalistic stimulation with brain connectivity measurements show that the default mode network contributes to information encoding (Simony et al., 2016), memory representations (Chen et al., 2017) and prediction of future events (Antony et al., 2021). Interestingly, it has been argued that the same network is also important for constructing emotional experiences (Satpute and Lindquist, 2019) and for the integration of the self with the social world (Yeshurun et al., 2021). Building upon this evidence, in a first experiment we explore how dynamic reconfigurations of brain networks (Fig. 1) explain changes in the subjectively reported affective experience during the viewing of a 2-hour long emotionally charged movie (Forrest Gump; Hanke et al., 2016).
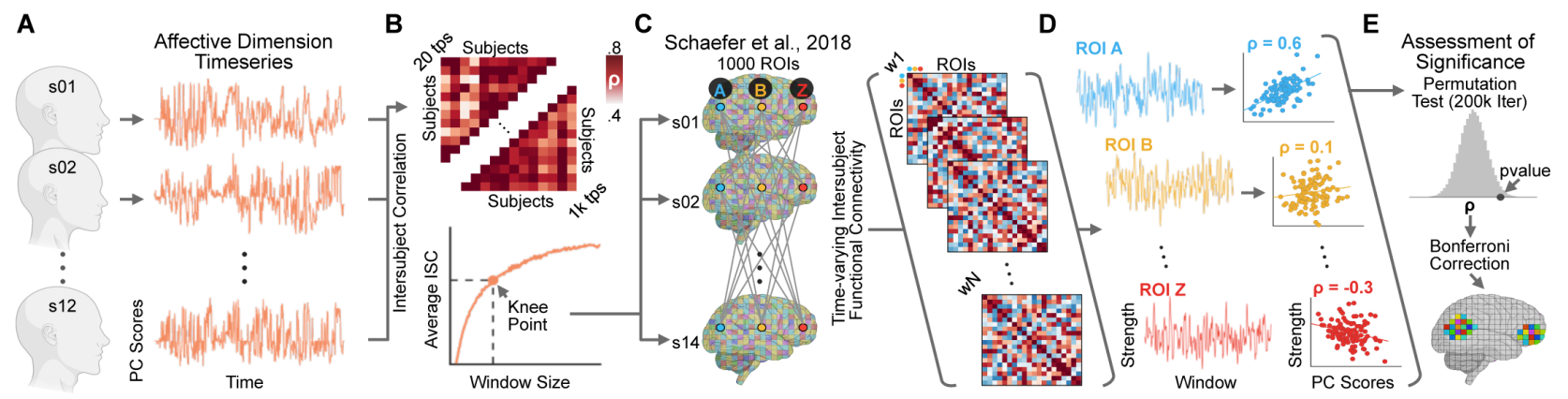

Figure 1 summarizes how we compute the association between affective dimensions extracted from behavioral ratings and timevarying connectivity strength of 1,000 brain regions. Each subject provided ratings of the affective experience during movie watching. We then applied principal component analysis to reveal single-subject affective dimension timeseries (polarity, complexity and intensity; panel A). Affective dimensions were correlated across subjects using a sliding window approach with multiple window size, ranging from 20 to 1,000 timepoints. Based on the tradeoff between window size and average intersubject correlation in behavioral ratings we selected the optimal width (i.e., knee point; panel B) for the subsequent estimation of brain connectivity dynamics. Brain activity was extracted from 1,000 regions of interest using Schaefer parcellation (2018) and time-varying intersubject functional correlation (tISFC) was computed following Simony et al., 2016 (panel C). For each brain area we estimated changes in connectivity strength on the complete graph and correlated (Spearman's $\rho$ ) the obtained timeseries with the timecourse of group-level affective dimensions (panel D). The assessment of significance was performed using a non-parametric permutation test and Bonferroni correction for multiple comparisons was then applied ( $\mathrm{n}=1,000$; critical p-value was 5.0e-5; panel $\mathbf{E})$.

Results demonstrate that pleasantness and intensity of the affective experience are associated with connectivity of default mode network and converge in the right temporo-parietal cortex.

We validate these results in a second experiment using emotional reports and brain data from independent participants watching a different live action movie (i.e., 101 Dalmatians). In addition, 
given the impact of analytical choices on brain imaging results (Botvinik-Nezer et al., 2020), we prove the generalizability of findings by preprocessing fMRI data using two alternative workflows. Lastly, in line with topographies revealed in high-order associative regions (Baldassano et al., 2017), our results demonstrate a chronotopic (Protopapa et al., 2019) organization of affect in temporoparietal and fronto-polar cortex. Here, distinct brain areas preferentially track changes in the emotional experience at different timescales, ranging from a few to several minutes. 


\section{Results}

Experiment 1: Single-Subject Affective Dimensions obtained from Behavioral Ratings. Affective states elicited by watching Forrest Gump are described by three orthogonal affective dimensions (Lettieri et al., 2019): (a) polarity (45\% of the explained variance) defines whether and to what extent the current experience is perceived as pleasant (negative scores) or unpleasant (positive scores); (b) complexity ( $24 \%$ of the explained variance) represents how much the affective state is associated with cognitive processes (positive scores), rather than being characterized by fast and automatic responses (negative scores); (c) intensity (16\% of the explained variance) denotes whether the experience is perceived as highly (high positive scores) or mildly (low positive scores) emotional. These three dimensions are consistent across all subjects $(\mathrm{p}<0.05)$ and explain approximately $85 \%$ of the variance in behavioral ratings. For polarity scores, median Spearman's correlation across subjects is $\rho=0.580$ (95\% CI: $0.560-0.615)$ with an interquartile range of $\rho=0.124$. For complexity scores, median Spearman's correlation across subjects is $\rho=0.437$ (95\% CI: $0.408-0.462)$ with an interquartile range of $\rho=0.124$. Lastly, for intensity ratings, median Spearman's correlation across subjects is $\rho=0.410$ (95\% CI: $0.391-0.423)$ with an interquartile range of $\rho=0.089$.

To estimate the association between time-varying intersubject functional correlation (tISFC) and changes in the reported affective experience, we first identified the optimal width of the moving window considering the intersubject correlation of affective dimensions. A tradeoff between the across-participants agreement and window width is reached at 273 timepoints $(9 \mathrm{~m}: 6 \mathrm{~s})$ for polarity, at 205 (6m:50s) for complexity and at $331(11 \mathrm{~m}: 2 \mathrm{~s})$ for intensity (for further details please refer to $f M R I$ Experiment: Intersubject Functional Correlation in Methods section). Therefore, affective dimension timeseries were down-sampled to these temporal resolutions and correlated with brain connectivity dynamics.

Experiment 1: Association between tISFC and Polarity of the Emotional Experience. Connectivity strength of four nodes of the default mode network is significantly associated with changes in the 
bioRxiv preprint doi: https://doi.org/10.1101/2020.06.06.137851; this version posted March 1, 2021. The copyright holder for this preprint (which was not certified by peer review) is the author/funder, who has granted bioRxiv a license to display the preprint in perpetuity. It is made available under aCC-BY-ND 4.0 International license.

polarity of the affective experience $\left(\mathrm{p}_{\mathrm{Bonf}}<0.05\right.$; Figure $2 \mathrm{~A}$ and $2 \mathrm{~B}$; Supplementary Table 1$)$. These four regions are the right temporoparietal junction, the right dorsal fronto-polar area, the right dorsomedial prefrontal cortex and the left precuneus. Connectivity of two other brain areas correlates with the perceived pleasantness of events: the right insula, which is part of the ventral attention network and the left middle occipital gyrus, a node of the dorsal attention network. Except for the left middle occipital gyrus, all other significant regions are negatively associated with polarity scores, meaning that the more unpleasant the experience is (i.e., positive scores), the weaker is the connectivity of default mode and ventral attention nodes. Instead, connectivity of the dorsal attention region increases for unpleasant events.
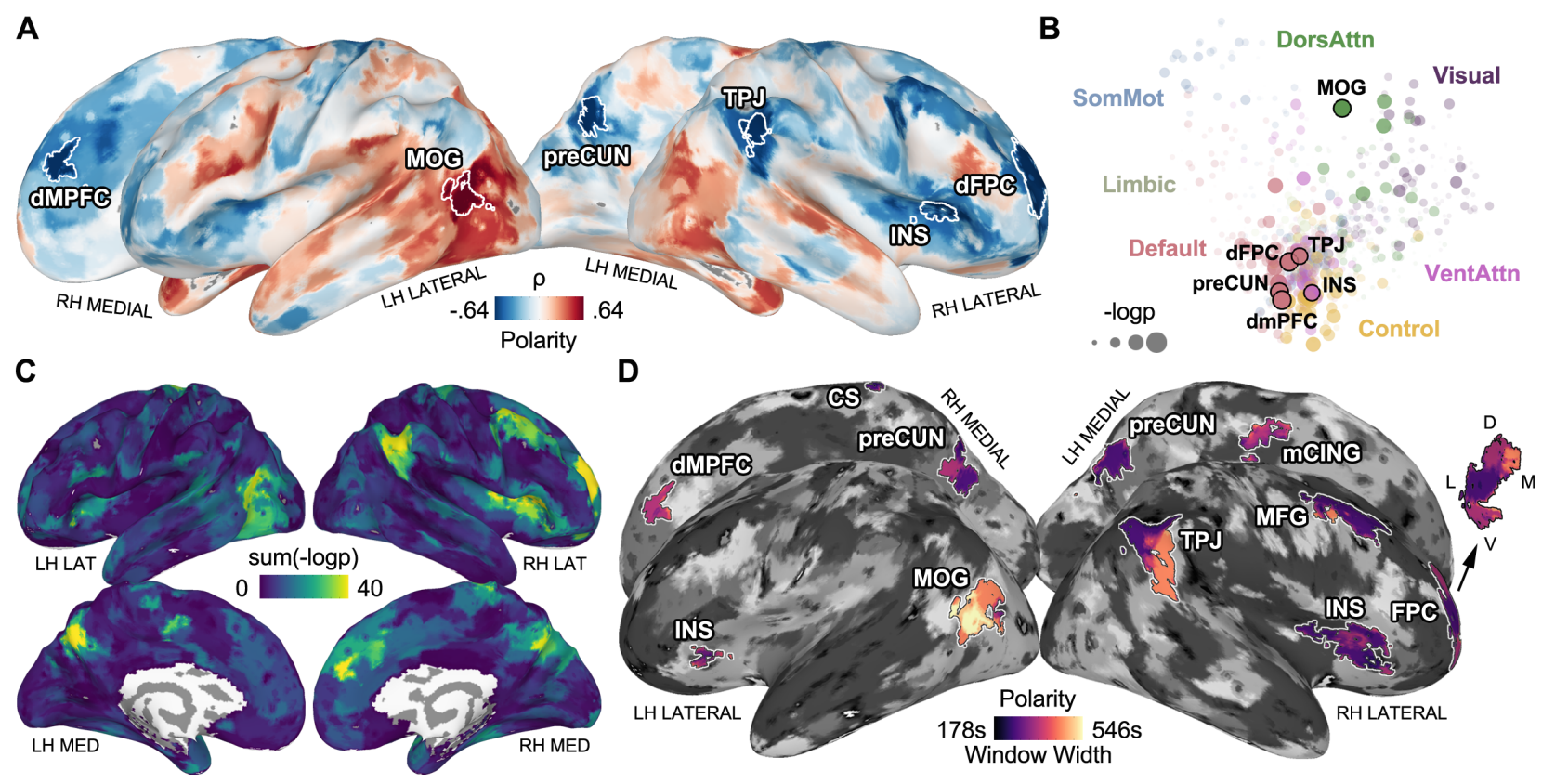

Figure 2. Panel A shows results for the correlation (Spearman's $\rho$ ) between tISFC strength for each brain region and the timecourse of polarity (red colors denote positive, whereas blue colors negative relationships). White outlines define regions surviving the Bonferroni correction for multiple comparisons ( $\mathrm{n}=1,000$ regions of interest; critical $\mathrm{p}$-value: $5.0 \mathrm{e}-5)$. In panel $\mathbf{B}$, the functional distance between brain regions is mapped using multidimensional scaling. Each brain area (i.e., dot) is color-coded depending on network membership, whereas its size and transparency are scaled according to the - $\log$ (pvalue) of the relationship with polarity. Of note, the triangular arrangement of brain regions follows the typical transitions between unimodal (i.e, visual and somatomotor) and transmodal (i.e., default mode and control) areas found in Margulies et al., 2016 using resting state connectivity. Panel $\mathbf{C}$ represents the association between tISFC strength of each brain region and polarity at all explored timescales (from 89 to 273 timepoints). Colors reflect the sum of $-\log$ (pvalue) across timescales, ranging from no involvement at any timescale (i.e., dark blue) to consistent recruitment across conditions (i.e., yellow). Lastly, panel $\mathbf{D}$ depicts the timescale at which connectivity dynamics are maximally associated with changes in polarity for each brain region. White outlines mark areas found to significantly encode polarity at one or more timescales (i.e., logical OR across timescales). $\mathrm{LH}=$ left hemisphere; $\mathrm{RH}=$ right hemisphere; $\mathrm{CS}=$ central sulcus; preCUN = precuneus; TPJ = temporoparietal junction; INS = insula; $\mathrm{dFPC}=$ dorsal fronto-polar cortex; $\mathrm{mCING}=$ mid cingulate cortex; $\mathrm{MFG}=$ middle frontal gyrus; $\mathrm{dmPFC}=$ dorsomedial prefrontal cortex; MOG = middle occipital gyrus; FPC = fronto-polar cortex; DorsAttn = dorsal attention network; VentAttn = ventral attention network; SomMot = somatomotor network; $\mathrm{D}=$ dorsal; $\mathrm{V}=$ ventral; $\mathrm{L}=$ lateral; $\mathrm{M}=$ medial. 
Experiment 1: Network Reconfiguration as function of the Polarity of Emotional Experiences. Figure 3 represents the correlation between significant regions and all the other brain areas for events rated either as highly pleasant (i.e., polarity scores below the $10^{\text {th }}$ percentile of the distribution) or highly unpleasant (i.e., polarity scores above the $90^{\text {th }}$ percentile). As compared to unpleasant events, when the experience is perceived as pleasant, right anterior nodes of the default mode network (i.e., dorsal fronto-polar area and dorsomedial prefrontal cortex) show stronger negative correlations with several nodes in the visual network (Figure $3 \mathrm{~B}$ and $3 \mathrm{C}$ ). Also, with changes in polarity, the connectivity between these regions and other default mode nodes remains substantially identical. Correlation between the left precuneus and visual areas becomes more negative for pleasant experiences as well (Figure 3D). Moreover, the left precuneus shows a strengthening of negative correlations with dorsal attention regions when the experience is rated as highly unpleasant. The vast majority of connections of the right temporoparietal junction are related to changes in polarity, with an increase in magnitude of both positive and negative correlations moving from unpleasant to pleasant events (Figure 3A). During unpleasant events the right insula is negatively correlated with specific default mode and somato-motor regions. The magnitude of this correlation decreases when subjects are exposed to pleasant events (Figure 3E). The opposite behavior is observed when considering the association between the right insula and areas belonging to the visual network - i.e., weakening of negative correlations for unpleasant as compared to pleasant events. Changes in polarity also modulate the relationship between the right insula and other ventral attention and control network regions. Lastly, for the left middle occipital gyrus, the correlation becomes more positive with other dorsal and ventral attention areas when the experience is unpleasant (Figure 3F). During pleasant events, instead, the coupling of this region with the visual network goes from negative to positive. 

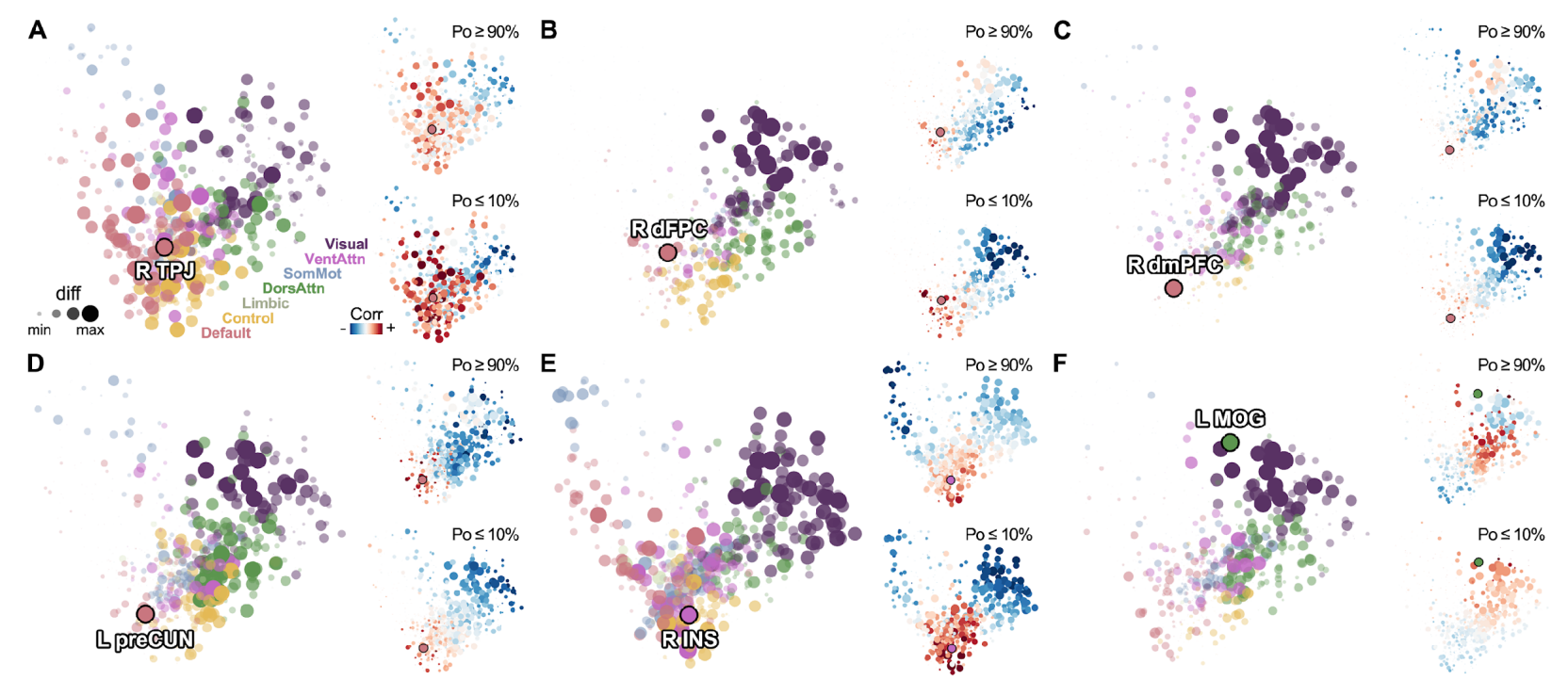

Figure 3 shows reconfigurations of functional connectivity during events rated either as highly pleasant (i.e., polarity scores below the $10^{\text {th }}$ percentile) or highly unpleasant (i.e., polarity scores above the $90^{\text {th }}$ percentile). Within each panel (A-F), functional distances of the 1,000 brain regions (i.e., dots) are expressed by the spatial arrangement in the multidimensional scaling space. In larger plots, regions are color-coded according to network membership and scaled in size and transparency based on the difference in correlation between the region of interest (i.e., black outlined dot) and the rest of the brain in pleasant and unpleasant events. Similarly, in smaller plots, size and transparency of each dot depends on the difference in correlation as a function of polarity values, whereas color indicates the correlation with the region of interest (red positive and blue negative relationships). VentAttn = ventral attention network; DorsAttn $=$ dorsal attention network; SomMot $=$ somatomotor network; $\mathrm{R}$ TPJ $=$ right temporoparietal junction; $\mathrm{R}$ dFPC $=$ right dorsal frontopolar cortex; $\mathrm{R} \mathrm{dmPFC} \mathrm{=} \mathrm{right} \mathrm{dorsomedial} \mathrm{prefrontal} \mathrm{cortex;} \mathrm{L}$ preCUN = left precuneus; $\mathrm{R}$ INS = right insula; $\mathrm{L}$ MOG $=$ left middle occipital gyrus.

Experiment 1: Chronotopic Maps of the Polarity of Emotional Experiences. We replicated the analysis of the relationship between polarity and brain connectivity dynamics at three alternative timescales (see Materials and Methods for further details): 89 timepoints (2m:58s), 143 timepoints (4m:46s) and 239 timepoints (7m:58s). The right dorsal fronto-polar area, the right insula and the left precuneus are associated with changes in polarity at all the explored timescales (see Figure $2 \mathrm{C}$ and Supplementary Figure 1). Also, connectivity of the right temporoparietal junction, the right dorsomedial prefrontal cortex and the right caudal middle frontal gyrus is significantly related to the pleasantness of the experience at three out of four timescales. Regions as the left middle occipital gyrus, the right precuneus and the right central sulcus track changes in polarity at two out of four time intervals, whereas the left insula and the left mid-cingulate sulcus at a single time window. For each of these regions, we report the preferred timescale for the processing of polarity in Figure 2D. Connectivity of the right insula, the right caudal middle frontal gyrus, the right central sulcus and the 
left precuneus preferentially encodes pleasantness of the events at shorter timescales ( $\sim 3$ minutes), whereas the right dorsomedial prefrontal cortex, the left insula and the left mid-cingulate sulcus at intermediate time windows ( $\sim 5$ minutes). The only brain area showing a marked preference for longer timescales is the left middle occipital gyrus (from $\sim 8$ to $\sim 9$ minutes). In addition, specific chronotopic maps (Protopapa et al., 2019) are revealed in the right temporoparietal junction, the right dorsal fronto-polar cortex and the right precuneus. Within the temporoparietal junction, connectivity of dorsocaudal territories is associated with polarity scores at shorter timescales ( $\sim 3$ minutes), while ventrorostral portions preferentially track pleasantness in $\sim 8$-minute segments. For the right precuneus, a rostrocaudal connectivity gradient suggests that, within this region, posterior territories preferentially encode polarity in 3-minute windows, while its anterior portions in 5-minute segments. Lastly, within the right dorsal fronto-polar area, connectivity is particularly associated with scores of the polarity component at shorter timescales ( $\sim 3$ minutes) in dorsolateral and ventrolateral territories, at intermediate intervals ( $\sim 5$ minutes) in the lateral region and at longer timescales $(\sim 8$ minutes $)$ in the dorsomedial part.

Experiment 1: Association between tISFC and Intensity of the Emotional Experience. Findings demonstrate that connectivity strength of ten brain regions is associated with the perceived intensity of the emotional experience ( $\mathrm{p}_{\mathrm{Bonf}}<0.05$; Figure 4A and 4B; Supplementary Table 2). Three of these brain areas - the right superior parietal lobule, the right intraparietal sulcus and the left postcentral sulcus - pertain to the dorsal attention network. Two regions belong, instead, to the ventral attention network: the right supramarginal gyrus and the ventral branch of the right precentral sulcus. Also, the caudal part of the right superior frontal gyrus and the pars orbitalis of the right inferior frontal gyrus, which are nodes of the control network, track changes in intensity. Lastly, connectivity of the anterior portion of the right temporoparietal junction, the right ventral fronto-polar area and the dorsal branch of the right precentral sulcus, respectively part of the default mode, the limbic and the somato-motor network, is associated to the intensity of the emotional experience. All these regions show a positive 
bioRxiv preprint doi: https://doi.org/10.1101/2020.06.06.137851; this version posted March 1, 2021. The copyright holder for this preprint (which was not certified by peer review) is the author/funder, who has granted bioRxiv a license to display the preprint in perpetuity. It is made available under aCC-BY-ND 4.0 International license.

relationship with the intensity dimension, meaning that the more intense the experience is, the stronger is the connectivity between these areas and the rest of the brain.
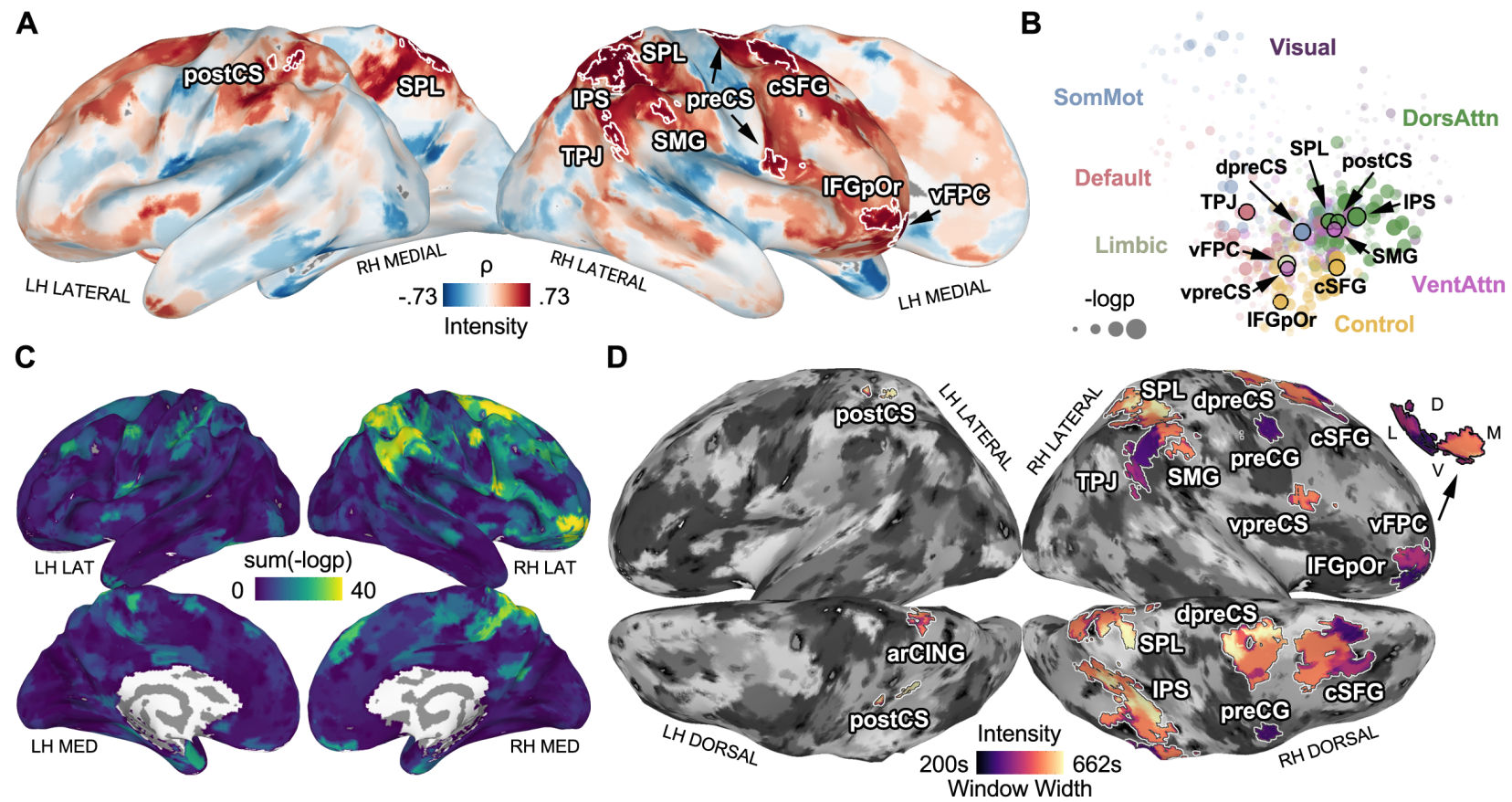

Figure 4. Panel A shows results for the correlation (Spearman's $\rho$ ) between tISFC strength for each brain region and the timecourse of intensity (red colors denote positive, whereas blue colors negative relationships). White outlines define regions surviving the Bonferroni correction for multiple comparisons ( $n=1,000$ regions of interest; critical p-value: $5.0 \mathrm{e}-5)$. In panel $\mathbf{B}$, the functional distance between brain regions is mapped using multidimensional scaling. Each brain area (i.e., dot) is color-coded depending on network membership, whereas its size and transparency are scaled according to the - $\log$ (pvalue) of the relationship with intensity. Panel $\mathbf{C}$ represents the association between tISFC strength of each brain region and intensity at all explored timescales (from 100 to 331 timepoints). Colors reflect the sum of $-\log$ (pvalue) across timescales, ranging from no involvement at any timescale (i.e., dark blue) to consistent recruitment across conditions (i.e., yellow). Lastly, panel $\mathbf{D}$ depicts the timescale at which connectivity dynamics are maximally associated with changes in intensity for each brain region. White outlines mark areas found to significantly encode intensity at one or more timescales (i.e., logical OR across timescales). $\mathrm{LH}=$ left hemisphere; $\mathrm{RH}=$ right hemisphere; postCS $=$ postcentral sulcus; SPL = superior parietal lobule; IPS = intraparietal sulcus; TPJ = temporoparietal junction; $\mathrm{SMG}=$ supramarginal gyrus; preCS $=$ precentral sulcus; $\mathrm{cSFG}=$ caudal superior frontal gyrus; IFGpOr $=$ pars orbitalis of the inferior frontal gyrus; $\mathrm{vFPC}=$ ventral frontopolar cortex; dpreCS $=$ dorsal branch of the precentral sulcus; vpreCS $=$ ventral branch of the precentral sulcus; DorsAttn $=$ dorsal attention network; VentAttn $=$ ventral attention network; SomMot $=$ somatomotor network; $\mathrm{D}=$ dorsal; $\mathrm{V}=$ ventral; $\mathrm{L}=$ lateral; $\mathrm{M}=$ medial.

\section{Experiment 1: Network Reconfiguration as function of the Intensity of Emotional Experiences. Figure}

5 and Supplementary Figure 2 detail reconfigurations of functional connectivity during events either perceived as highly emotional (i.e., intensity scores above the $90^{\text {th }}$ percentile of the distribution) or rated as having modest impact (i.e., intensity scores below the $10^{\text {th }}$ percentile). As compared to low intensity events, those rated as highly emotional are characterized by a strengthening of the negative relationship between nodes of the right dorsal attention network (Figure 5A and 5F and 
Supplementary Figure 2A) and default mode regions, as well as by an increase in the magnitude of the positive correlation between dorsal attention regions and control and ventral attention nodes. When the experience is rated as highly intense, regions of the ventral attention network, as the inferior branch of the precentral sulcus, become more negatively coupled with visual and default mode regions (Figure 5C and Supplementary Figure 2C). At the same time, these brain areas strengthen their positive relationship with control and dorsal attention nodes. Moving from low to high emotional events, the anterior portion of the right temporoparietal junction increases the within-network positive correlation with other default mode regions and decreases the anti-correlation with dorsal attention areas (Figure 5B). Also, this brain region is negatively related to the somato-motor network during low intensity events, whereas it becomes positively associated with the same areas when the experience is perceived as highly intense. Connectivity of the ventral fronto-polar area shows a clear switch in the coupling with default mode regions (Figure 5D): during low intensity events, this limbic area demonstrates a positive relationship with default mode nodes, which then becomes negative when the experience is rated as highly emotional. In addition, moving from low to high intensity events, the ventral fronto-polar region reinforces the positive relationship with the control network. Similarly, also the pars orbitalis of the right inferior frontal gyrus shows a negative correlation with the default mode and the somato-motor network during intense events (Figure 5E). This relationship is then reversed (i.e., becomes positive) when considering low intensity experiences. Lastly, this control network area becomes decoupled from visual nodes during high, as compared to low, intensity events. 
A

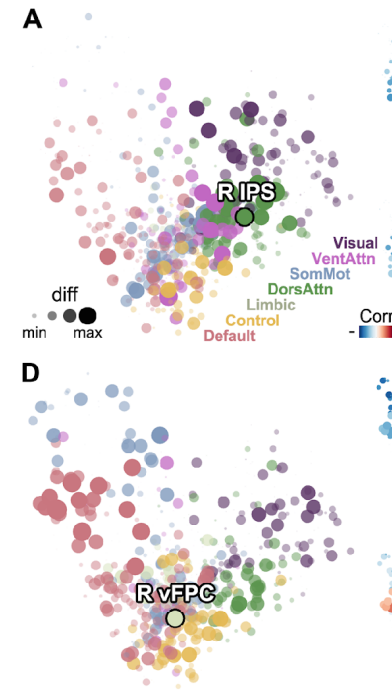

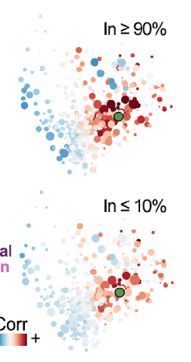

B
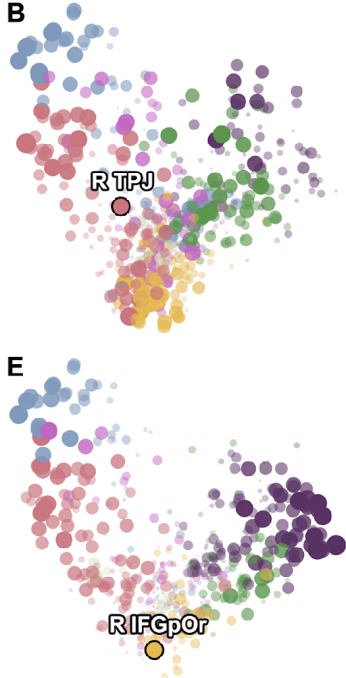
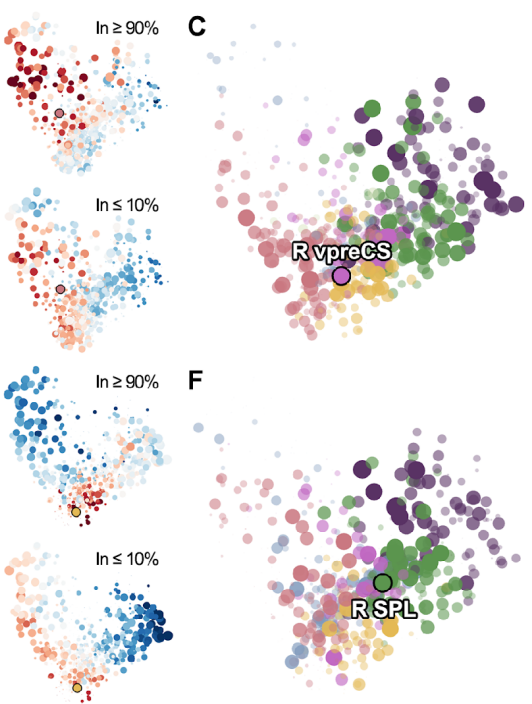

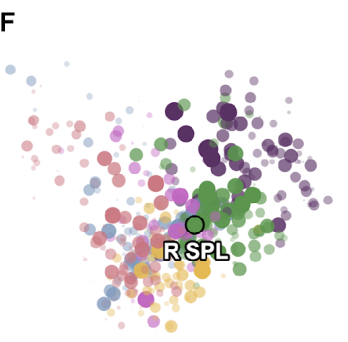

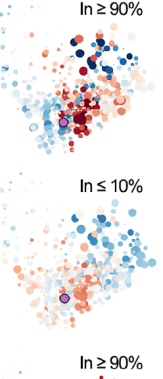

$\ln \geq 90 \%$
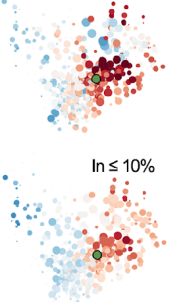

Figure 5 shows reconfigurations of functional connectivity during events perceived as highly emotional (i.e., intensity scores above the $90^{\text {th }}$ percentile) or rated as having modest impact (i.e., intensity scores below the $10^{\text {th }}$ percentile). Within each panel (A-F), functional distances of the 1,000 brain regions (i.e., dots) are expressed by the spatial arrangement in the multidimensional scaling space. In larger plots, regions are color-coded according to network membership and scaled in size and transparency based on the difference in correlation between the region of interest (i.e., black outlined dot) and the rest of the brain in highly and mildly emotional events. Similarly, in smaller plots, size and transparency of each dot depends on the difference in correlation as a function of intensity values, whereas color indicates the correlation with the region of interest (red positive and blue negative relationships). VentAttn = ventral attention network; DorsAttn = dorsal attention network; SomMot $=$ somatomotor network; R TPJ = right temporoparietal junction; R IPS = right intraparietal sulcus; $\mathrm{R}$ vpreCS $=$ right ventral branch of the precentral sulcus; $\mathrm{R} v \mathrm{FPC}=$ right ventral fronto-polar cortex; R IFGpOr = pars orbitalis of right inferior frontal gyrus; R SPL = right superior parietal lobule.

Experiment 1: Chronotopic Maps of the Intensity of Emotional Experiences. As in the case of the polarity dimension, we replicated the analysis of correlation between brain connectivity and intensity scores at three additional timescales. Changes in the slope of intersubject agreement in intensity ratings were detected at 100 (3m:20s), 163 (5m:26s) and 247 timepoints (8m:14s). The right ventral fronto-polar area and the right caudal superior frontal gyrus are associated with changes in intensity at all explored timescales (see Figure 4C and Supplementary Figure 3). Connectivity of the right temporoparietal junction, the right precentral gyrus, the ventral branch of the right precentral sulcus, the pars orbitalis of the right inferior frontal gyrus and the right superior parietal lobule encodes changes in the intensity of the emotional experience at three out of four timescales. The right intraparietal sulcus, the right supramarginal gyrus and the dorsal branch of the right precentral sulcus are, instead, significantly associated with intensity scores in half of the considered timescales. Lastly, 
connectivity strength of the right lateral orbitofrontal cortex and of the left postcentral sulcus significantly correlates with intensity at a single time range.

For all these regions, we report the preferred timescale for the processing of intensity of the emotional experience in Figure 4D. Similarly to the polarity results, some regions, as the right temporoparietal junction, the right caudal superior frontal sulcus and a large cluster including the right lateral orbitofrontal cortex, the pars orbitalis of the right inferior frontal gyrus and the right ventral frontopolar cortex, show timescale topographies. For the right temporoparietal junction, connectivity of the ventral part preferentially encodes intensity in $\sim 5$-minute segments (i.e., 163 timepoints), whereas its rostro-dorsal portion favors shorter timescales ( $\sim 3$ minutes). Within the right inferior parietal lobule, moving from the angular gyrus to the posterior region of the supramarginal, we find that the association between connectivity and intensity scores peaks at longer timescales ( $\sim 8$ minutes). As far as the right caudal superior frontal gyrus is concerned, the connectivity of the rostro-medial part preferentially represents the intensity of the experience at shorter timescales $(\sim 3$ minutes), whereas the rostro-lateral portion achieves the highest significance considering $\sim 5$-minute windows. These two regions are separated by a larger cluster preferentially encoding intensity at longer timescales $(\sim 8$ minutes). In the right ventral prefrontal territories, the connectivity of a large cluster is maximally associated with intensity scores at three distinct timescales: the lateral orbitofrontal cortex shows a preference for shorter time intervals ( $\sim 3$ minutes), the pars orbitalis for intermediate timescales $(\sim 5$ minutes) and the ventral fronto-polar area for longer segments ( $\sim 8$ minutes).

In addition, results demonstrate that the association between connectivity and intensity of the emotional experience is maximal at longer timescales $(\sim 11$ and $\sim 8$ minutes $)$ for the right superior parietal and intraparietal regions, for the dorsal and ventral branches of the precentral sulcus and for the left postcentral sulcus. Instead, the right precentral gyrus demonstrates its preference for shorter timescales $(\sim 3$ minutes $)$. 
Experiment 1: Association between tISFC and Complexity of Emotional Experience. Changes in the complexity dimension are not associated with the connectivity of any brain region when considering the optimal window width of 205 timepoints (6m:50s; Supplementary Figure 4D). Nevertheless, results show that connectivity strength of the right mid-cingulate cortex, the right inferior temporal gyrus and the left rostral superior frontal gyrus tracks changes in complexity at shorter timescales (i.e., 88 and 109 timepoints, respectively 2m:56s and 3m:38s; Supplementary Figure 4A-B).

Experiment 2: Validation of the relationship between tISFC and Affective Dimensions. To test the reliability and generalizability of results obtained from Forrest Gump (i.e., exploratory dataset) we collected and analyzed emotion dimension ratings of the live action movie 101 Dalmatians (i.e., validation dataset). We first downsampled polarity and intensity reports to match the fMRI resolution (1,614 timepoints) and correlated the obtained timeseries across subjects. For polarity, median Spearman's correlation across subjects is $\rho=0.487$ (95\% CI: $0.452-0.530)$ with an interquartile range of $\rho=0.293$. Also, the $97.1 \%$ (204 out of 210 ) of correlations across all possible pairings of subjects reach the statistical significance after correction for multiple comparisons ( $\left.\mathrm{p}_{\text {Bonf }}<0.05\right)$. For intensity, instead, median correlation is $\rho=0.229$ (95\% CI: $0.203-0.245)$ with an interquartile range of $\rho=0.184$ and the $88.6 \%$ (186 out of 210 ) of correlation values are significant ( $\left.\mathrm{p}_{\mathrm{Bonf}}<0.05\right)$. As in experiment 1, we downsampled affective dimension timeseries based on the estimate of the optimal window size (polarity: 67 timepoints; intensity: 37 timepoints). Group averaged polarity and intensity ratings were then used to explain the time-varying brain connectivity in independent subjects watching the same movie. We restricted these analyses to regions significantly encoding affective dynamics in the exploratory dataset (i.e., experiment 1) and applied correction for multiple comparisons based on the number of regions of interest.

Results show that both polarity and intensity ratings are associated with connectivity strength of the right temporo-parietal junction $\left(\mathrm{p}_{\text {Bonf }}<0.05\right.$ over 37 regions for polarity and $\mathrm{p}_{\text {Bonf }}<0.05$ over 35 regions for intensity; Figure 6A and B). Importantly, this finding does not depend on the fMRI data 
bioRxiv preprint doi: https://doi.org/10.1101/2020.06.06.137851; this version posted March 1, 2021. The copyright holder for this preprint (which was not certified by peer review) is the author/funder, who has granted bioRxiv a license to display the preprint in perpetuity. It is made available under aCC-BY-ND 4.0 International license.

preprocessing pipeline (Figure 6D) and the relationship between affect and brain connectivity is interpreted as in the exploratory dataset: the more unpleasant and less intense the experience is, the weaker is the connectivity between this default mode network area and the rest of the brain (Figure $6 \mathrm{E}$ and $\mathrm{F})$. To further prove the similarity between results obtained from the two experiments, we show that regions of overlap between maps of polarity and intensity in the exploratory dataset (Figure 6C) also represent the timecourse of these two affective dimensions in the validation dataset $\left(\mathrm{p}_{\text {Bonf }}<\right.$ 0.05), regardless of the preprocessing workflow.

In addition, connectivity strength of a default mode network region within the right dorsal frontopolar cortex is negatively associated with changes in polarity $\left(\mathrm{p}_{\mathrm{Bonf}}<0.05\right.$ for workflow $\mathrm{A}$ and $\mathrm{p}<$ 0.05 for workflow B; Figure 6D and E). Another parcel within the same fronto-polar area, though pertaining to the control network, correlates with the pleasantness of the experience $\left(\mathrm{p}_{\text {Bonf }}<0.05\right.$ for both workflow A and B; Figure 6D and E). Lastly, two regions of the control network within the right middle frontal gyrus and the left central sulcus (belonging to the somato-motor network) are associated with polarity scores.
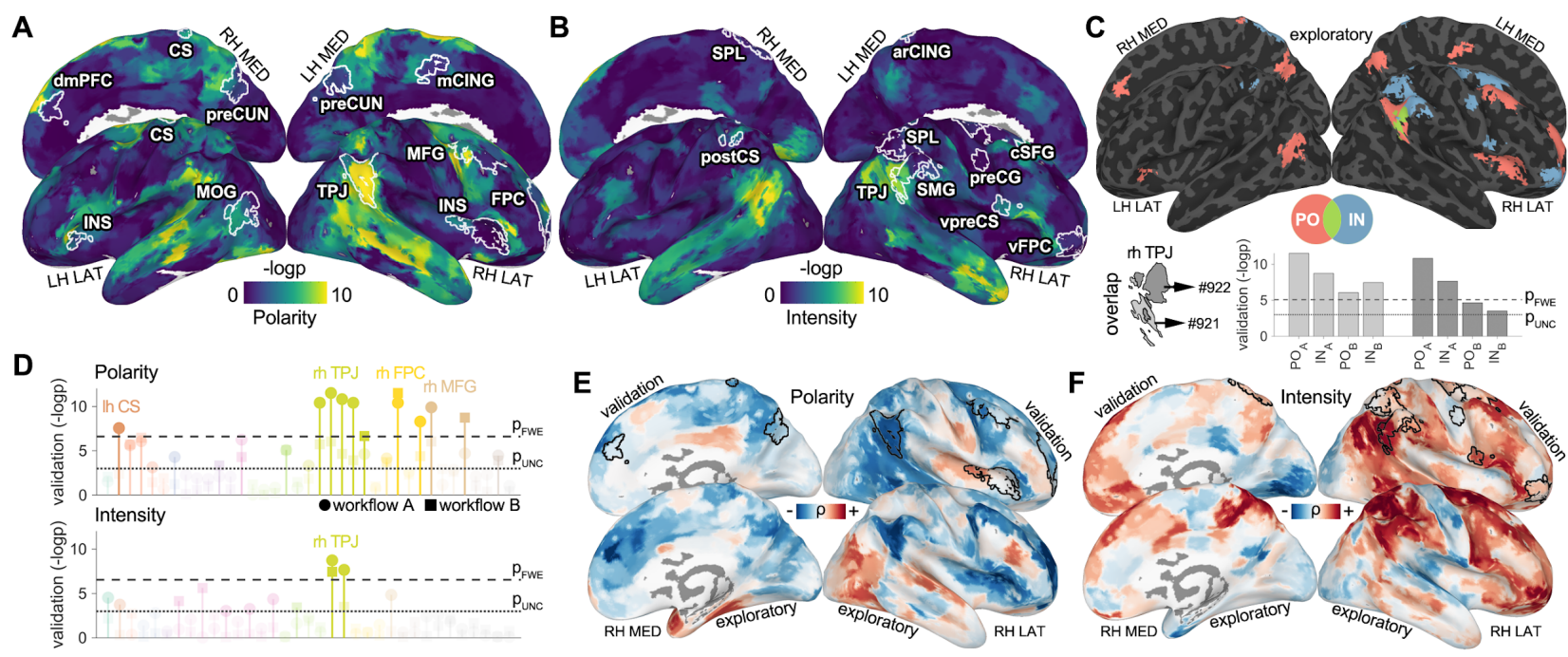

Figure 6 shows the validation of the relationship between tISFC and affective dimensions. Panels $\mathbf{A}$ and $\mathbf{B}$ depict unthresholded maps of the association between time-varying brain connectivity (workflow A) and ratings of polarity (A) and intensity (B) in the validation dataset (i.e., 101 Dalmatians; experiment 2). White outlines indicate significant regions in the exploratory dataset (i.e., Forrest Gump; experiment 1). Panel C shows the conjunction analysis between maps of polarity (i.e., red), intensity (i.e., blue) and their overlap (i.e., green) in the exploratory dataset. The bar chart demonstrates that connectivity of the overlap region \#921 (Schaefer et al., 2018) within the right temporo-parietal junction encodes both affective dimensions in the validation dataset, regardless of the preprocessing applied to fMRI data. The Manhattan plot in panel D summarizes the significance of the association between IISFC and affective dimensions across all regions obtained from experiment 1 . Connectivity of the right temporo-parietal 
bioRxiv preprint doi: https://doi.org/10.1101/2020.06.06.137851; this version posted March 1, 2021. The copyright holder for this preprint (which was not certified by peer review) is the author/funder, who has granted bioRxiv a license to display the preprint in perpetuity. It is made available under aCC-BY-ND 4.0 International license.

junction, a relevant node of the default mode network, is associated with changes in polarity and intensity, irrespective of the preprocessing pipeline $\left(\mathrm{p}_{\mathrm{FWE}}<0.05\right)$. Polarity is also represented by connectivity of other default mode, control and somato-motor regions, such as the right dorsal fronto-polar cortex, the right middle frontal gyrus and the left central sulcus. Panel $\mathbf{E}$ and $\mathbf{F}$ allow for a direct comparison between the exploratory (top rows) and the validation (bottom rows) datasets in terms of Spearman's correlation between brain connectivity and the timecourse of polarity $(\mathbf{E})$ and intensity $(\mathbf{F})$. Black outlines indicate regions of interest extracted from experiment 1 . 


\section{Discussion}

In the current study, we combined real-time reports of the affective experience with recordings of fMRI activity during naturalistic stimulation, to reveal how the brain encodes the stream of affect. Over two experiments, we collected approximately 45 hours of behavioral reports and 38 hours of brain imaging data. In experiment 1 (i.e., exploratory dataset), ratings of emotion categories provided by Italian participants during the watching of Forrest Gump were used to obtain timeseries of emotion dimensions (i.e., polarity, complexity and intensity) and to explain brain connectivity dynamics in German individuals. A second movie, 101 Dalmatians, was used to test the reliability and generalizability of results obtained from the exploratory dataset (i.e., experiment 2): moment-bymoment scores of the pleasantness and intensity of the emotional experience were correlated with changes in connectivity of brain regions identified in experiment 1. Also, fMRI data were preprocessed according to two alternative workflows. Therefore, our study protocol ensures that findings cannot be merely ascribed to (1) the selection of a specific movie, (2) the characteristics of individuals participating in the research, (3) the method used to collect behavioral reports, (4) the pipeline adopted to preprocess fMRI data.

Results show that connectivity strength of the right temporoparietal junction and of the right dorsal fronto-polar cortex, both pertaining to the default mode network, is positively associated with the pleasantness of the affective experience. Nodes of the control network, such as the right middle frontal gyrus, also encodes polarity during movie watching. In addition, polarity and intensity converge in the right temporo-parietal cortex, as connectivity of this region also tracks changes in the emotional impact over time. Of note, together with the fronto-polar cortex, the right temporo-parietal junction represents the dynamics of affect in a chronotopic manner, meaning that adjacent portions of these areas preferentially map the stream of affect at different time intervals, ranging from a few to several minutes.

In everyday life, affect varies as a function of context, expectations and personal motivations. These features are intrinsically related to time and their complex relationship is hardly recreated in the 
laboratory setting. Thus, researchers are confronted with a difficult challenge when they seek to investigate how emotional reports relate to brain activity over time. This is presumably one of the reasons for the paucity of neuroimaging studies on the temporal characteristics of affect.

To elicit a variety of affective states, track their evolution in time and maintain a relatively high ecological validity, an optimal solution may be represented by movie watching. As a matter of fact, movies produce strong affective responses over a few hours and mimic everyday life situations with an alternation of sudden and predictable events (Schaefer et al., 2010). Also, changes in affect induced by movies are based on the rich contextual information provided by the plot and are related to the personal expectations of the viewer.

Building upon this, we recorded brain activity and moment-by-moment affective ratings during the watching of two movies, and demonstrated that changes in the subjective emotional experience are explained by brain connectivity dynamics. Over the last decade, researchers have emphasized the importance of moving from a region- to a network-based approach when studying the neural correlates of emotion (Lindquist and Barrett, 2012; Raz et al., 2016; Kragel et al., 2016; Pessoa, 2017). As a matter of fact, changes in affect are associated with modifications in bodily responses (e.g., autonomic, musculoskeletal) and mental processes (e.g., memory, decision making), which is likely reflected in a distributed functional reorganization of brain modules (Pessoa, 2017). In this regard, among large-scale brain networks, the default mode network is thought to play a crucial role for the construction and representation of emotions (Satpute and Lindquist, 2019). Satpute and Lindquist claim that the conceptualization of emotion categories as patterns of physiological and behavioral responses requires the ability to abstract concrete features (e.g., a smile, the increase in respiration rate) to mental categories (e.g., joy, fear) and that the default mode network is the best candidate to carry out such a computation. Interestingly, the same network also supports processes of mind wandering, it is engaged during internally focused states (Raichle, 2015) and it is involved in the combination of sensory inputs coming from internal organs (Kleckner et al., 2017). Our findings support the hypothesis of the central role of the default mode network in the processing of affect, as 
we demonstrate that its connectivity tracks changes in the subjective judgment of pleasantness and emotional intensity during movie watching. This task requires, on the one hand, theory of mind and perspective taking to understand and process emotions, expectations and beliefs of movie characters (e.g., whether the protagonist feels in a positive or negative manner) and, on the other hand, that viewers provide subjective descriptions of their own experience (e.g., how one feels about what happens to the protagonist). Importantly, these third- and first-person computations can be both carried out by the default mode (Schilbach et al., 2008). Our results are also in line with reports that highlighted the role of this network in high paranoia traits or depressed patients, in whom negative rumination is intense and related to resting state hyperconnectivity or to an increased across-subjects synchronization (Finn et al., 2018). Indeed, here we demonstrate that when healthy subjects experience negative situations, the connectivity of the default mode network with the rest of the brain is actually reduced, whereas it is increased when experiencing positive emotions. Similarly, a recent longitudinal study in a single healthy participant demonstrated that connectivity of the default mode is directly related to positive mood (Mirchi et al., 2019).

Furthermore, we show across two experiments that connectivity of control network nodes is related to the polarity of affect. The control network is claimed to regulate the activity of the attention and the default mode networks, to mediate between potentially conflicting evidence obtained from inner and outer processes (Gao and Lin, 2012) and to be involved in empathy and theory of mind (Bzdok et al., 2012). In the experience of emotions, it has been hypothesized that, together with the default mode, the control network is essential in the construction and regulation of the perceived state (Lindquist and Barrett, 2012).

When we look closely at nodes encoding affective dimensions, we notice that connectivity strength of the right dorsal fronto-polar cortex tracks changes in the perceived pleasantness of the emotional experience. Indeed, there is a general consensus on the involvement of the medial and lateral frontopolar cortex in social cognition abilities and mood (Shamay-Tsoory et al., 2005; Mirchi et al., 2019). Further, connectivity dynamics of the right temporoparietal junction represents both polarity and 
intensity. This cortical area is one of the core regions of the social brain, as it contributes to theory of mind (Saxe and Kanwisher, 2003), emotion regulation (Kohn et al., 2014; Etkin et al., 2015) and empathy (Lamm et al., 2007). In addition, within the right temporo-parietal cortex we recently uncovered the existence of orthogonal and spatially overlapping gradients representing emotion dimensions (Lettieri et al., 2019). In our previous study, we hypothesized that the right temporoparietal junction could also act as a central node of a distributed network, in which several regions contribute with distinct computations to the rising of emotions. Indeed, here we confirm our prediction by demonstrating that connectivity between this region and other cortical areas tracks multiple descriptions of affective states over time.

In experiment 1 , we also characterize the preferred timescale at which the connectivity of distinct brain regions represents specific descriptions of affect. Previous studies on memory and narrative comprehension demonstrate that the brain splits the experience at multiple timescales following a hierarchy, in which sensory regions extract information in short segments, whereas high-order areas preferentially encode longer events (Simony et al., 2016; Baldassano et al., 2017). We show that the same happens for the processing of affect, with chronotopic maps (Protopapa et al., 2019) representing the temporal dynamics of the emotional experience at specific timescales. As compared to studies on narrative comprehension and memory, our results highlight relatively slow dynamics, approximately between 3 and 11 minutes. This likely reflects the fact that changes in affect during movie watching are associated with the processing of longer movie excerpts, which are, in turn, characterized by temporal dynamics similar to those of the main sections of the plot. In addition, regions encoding affect are mainly high-order transmodal cortical areas (e.g., angular gyrus, precuneus), which are tuned to longer timescales (Baldassano et al., 2017). Interestingly, the right temporoparietal junction and the right fronto-polar cortex show peculiar topographies when considering the preferred timescale. Specifically, for polarity, the timescale increases moving from dorsocaudal to ventrorostral territories. For intensity, instead, changes occurring at shorter time intervals are represented by connectivity of ventrocaudal portions, whereas those relative to longer 
timescales are mapped in rostrodorsal areas. The right fronto-polar cortex, instead, has a patchier organization, with no clear evidence of linear gradients for the coding of affect at multiple timescales. Our work is not immune to some criticisms. Firstly, we focused on brain and affective dynamics in the range of a few to several minutes. As a matter of fact, the temporal resolution of fMRI is suited to capture relatively slower brain dynamics, whereas faster affective reactions may be better studied with other techniques. Secondly, we assessed the chronotopic organization of affect in the first experiment only, as this analysis requires the exploration of a wide range of temporal dynamics (i.e., from a few to tens of minutes). This was ensured by the movie we selected for experiment 1 (i.e., Forrest Gump), as it lasts twice as long as the film employed in experiment 2 (i.e., 101 Dalmatians). Lastly, as subcortical regions are not present in the original Schaefer (2018) parcellation, our analysis does not include them. These structures, however, typically demonstrate much faster dynamics as compared to those investigated here (e.g., Garvert et al., 2014).

In conclusion, here we demonstrate that brain connectivity dynamics track changes in subjective reports of the affective experience. Specifically, the right temporo-parietal and the dorsal fronto-polar cortex, two nodes of the default mode network, represent the pleasantness of the experience. Interestingly, polarity and intensity converge into the right temporoparietal cortex, as connectivity of this region tracks the emotional impact as well. Further, within the right temporo-parietal junction and the right fronto-polar cortex, the stream of affect is represented at multiple timescales following chronotopic connectivity maps. Altogether, these findings reveal that the human brain represents changes in affect through connectivity of the default mode network and that the timescale of emotional experiences is topographically mapped. 


\section{Materials and Methods}

Experiment 1: Behavioral Study - Participants. Twelve Italian native speakers (5F; mean age 26.6 years, range 24-34) provided ratings of their moment-by-moment emotional experience while watching the Forrest Gump movie (R. Zemeckis, Paramount Pictures, 1994). All participants gave their written informed consent to take part in the study after risks and procedures had been explained. They retained the right to withdraw at any time and received a small monetary compensation for their participation. All subjects were clinically healthy and had no history of any neurological or psychiatric condition. They also had normal hearing, normal or corrected to normal vision and reported no history of drugs or alcohol abuse. The local Ethical Review Board approved the experimental protocol and procedures (CEAVNO: Comitato Etico Area Vasta Nord Ovest; Protocol No. 1485/2017) and the study was conducted in accordance with the Declaration of Helsinki.

Experiment 1: Behavioral Study - Stimuli and Experimental Paradigm. Behavioral data have been reported previously (see Lettieri et al., 2019 for details) and made freely available at https://github.com/psychoinformatics-de/studyforrest-data-perceivedemotions. In brief, subjects sat comfortably in a silent room facing a 24" Dell ${ }^{\mathrm{TM}}$ screen, wore headphones (Sennheiser ${ }^{\mathrm{TM}}$ HD201; 21-18,000 Hz; Maximum SPL 108 dB), and were presented with an Italian dubbed and edited version of Forrest Gump. All participants had not watched the movie in the period of time of at least one year before the experiment. The movie was split into 8 movie segments ranging from 11 to 18 minutes, so to adhere to the fMRI protocol described in Hanke et al., 2016. To collect behavioral reports of the affective experience, subjects were asked to report moment-by-moment (i.e., 10Hz sampling rate) the type and intensity of their inner emotional state using six categories, according to the basic emotion theory (Ekman, 1992): happiness, surprise, fear, sadness, anger and disgust. Moreover, as the complexity of the experience elicited by Forrest Gump cannot be merely reduced to six emotions (Lettieri et al., 2019), we asked subjects not to limit their report to binary choices and use as many combinations of categories as they want, to best describe how they felt at each moment during the 
movie. This, together with the possibility to indicate the contribution (i.e., intensity) of each category to the experienced affective state, ensured that different instances of emotions are captured by our behavioral reports.

For each subject, the overall duration of the behavioral data acquisition was 120 minutes $(72,000$ timepoints). Stimulus presentation and recording of the responses were implemented in Matlab (R2016b; MathWorks Inc., Natick, MA, USA) and Psychtoolbox v3.0.14 (Kleiner et al., 2007).

Experiment 1: Behavioral Study - Single-Subject Affective Dimensions. Starting from categorical descriptions one can obtain the underlying dimensions using factor analysis or principal components. Studies on perception demonstrated that such dimensions are preferentially encoded in topographic maps (Harvey et al., 2013) and this may be the case also for the correlates of affective experiences (Lettieri et al., 2019). For this reason, by using principal component analysis (PCA) and procrustes rotation, we derived in each subject the timecourse of the affective dimensions explaining behavioral reports of the emotional experience. In brief, timeseries representing the emotional experience of each subject were downsampled to 2-second resolution (3,600 timepoints), matching the temporal characteristics of the fMRI acquisition. Data were then averaged across subjects and PCA was used to reveal group-level affective dimensions. PCA was also performed on single-subject data and the results were aligned to best approximate group-level dimensions using procrustes orthogonal linear transformation (reflection and rotation only). We considered the rotated scores of principal components as single-subject affective dimensions and used these data to inform subsequent fMRI analyses. To assess the agreement across subjects in affective dimensions, we also computed pairwise Spearman's correlation and its significance (see Lettieri et al., 2019 for further details).

Experiment 1: fMRI Study - Participants. Brain activity elicited by the watching of Forrest Gump was obtained from the studyforrest project - phase II (http://studyforrest.org). Brain data comprise recordings of fourteen healthy German subjects (6F; mean age 29.4 years, range 20-40 years), 
instructed to simply inhibit any movement and enjoy the movie. Similarly to the behavioral part of the study, the fMRI acquisition lasted two hours and was split in eight runs (3T Philips Achieva scanner; 32 channels head coil; gradient recall echo-echo planar imaging; 2000ms repetition time, $30 \mathrm{~ms}$ echo time, $90^{\circ}$ flip angle, $3 \mathrm{~mm}$ isotropic voxel, $240 \mathrm{~mm}$ field of view). Together with the fMRI data, 3D T1w high-resolution anatomical images were also acquired. For further details regarding data acquisition and protocol please refer to Hanke et al., 2016.

Experiment 1: fMRI Study - Data Pre-processing. AFNI v.17.2.00 (Cox, 1996) and ANTs (Avants et al., 2009) were used to preprocess the MRI data. For each subject, structural images were first skullstripped (antsBrainExtraction.sh) and transformed to match the MNI152 template using non-linear registration (3dQwarp). Functional data were corrected for intensity spikes (3dDespike), adjusted for slice timing acquisition ( $3 d T$ shift) and corrected for head motion (3dvolreg) by computing the displacement between each volume and the most stable timepoint of each run (i.e., the one showing smallest framewise displacement values; Power et al., 2012). Afterwards, the align_epi_anat.py and $3 d Q$ warp software were used to estimate the co-registration between functional and structural data, as well as to correct for phase distortion by allowing non-linear deformations in the y-direction (i.e., phase acquisition direction) only. Linear (i.e., motion correction, co-registration) and non-linear (i.e., phase distortion and MNI152 registration) transformations were then concatenated and applied (3dNwarpApply) to the functional data, so to obtain standard-space single-subject timeseries with a single interpolation step (i.e., sinc interpolation method), maintaining also the original spatial resolution (3mm isotropic voxel). In addition, data were iteratively smoothed until $6 \mathrm{~mm}$ full-width at half maximum level was reached ( $3 d B l u r T o F W H M)$ and rescaled $(3 d c a l c)$ so that changes in activity were expressed as percentage with respect to the average voxel intensity in time (3dTstat). Lastly, we used 3dDeconvolve to regress out from brain activity signal drifts (polort in 3dDeconvolve) and physiological confounds following the recommendations by Ciric et al., 2017. Therefore, we used a model with 36 regressors of no interest to reduce the possibility that results of intersubject functional 
correlation could be explained by factors other than neurovascular coupling. Specifically, we created (Atropos; 3dmask_tool) binary masks of the white matter (WM) and cerebrospinal fluid (CSF) and extracted the timeseries of the average WM and CSF activity. Global signal (GS), which is the average activity across all voxels, was also calculated. For each of the three obtained timeseries (i.e., GS, WM, CSF), we computed their temporal derivatives, the quadratic terms and squares of derivatives, resulting in 12 regressors. The remaining 24 comprised the six head motion parameters estimated with $3 d v o l r e g$, their temporal derivatives, their quadratic terms and the squares of derivatives. All regressors of no interest were then de-trended using the same degree of polynomial (polort) employed in 3dDeconvolve to remove slow drifts from voxels activity. The model was then fitted in brain activity using a mass-univariate general linear model. The residuals of this fitting were considered the single-subject brain activity associated with the watching of Forrest Gump and used in subsequent analyses.

Experiment 1: fMRI Study - Intersubject Functional Correlation. The intersubject functional correlation (ISFC) method measures brain inter-regional covariance induced by stimulation, as it computes the correlations between signal change of a specific brain region in one subject with activity of all other regions in other subjects (Simony et al., 2016). When compared to other measures of functional connectivity, ISFC demonstrates higher signal-to-noise ratio as it inherently filters out activity not related to stimulus processing and artifacts not correlated across brains. In addition, similarly to other functional connectivity methods, ISFC can be used to study dynamic reconfigurations of brain networks using a moving-window approach. We estimated the static and time-varying ISFC (tISFC) associated with the watching of Forrest Gump using custom MATLAB scripts that made freely available at https://osf.io/v8r9w/?view_only=6a8ac3e3c6ba43d0a205146b4975ebb4.

As a first step, we selected the atlas provided by Schaefer and colleagues (2018) and parcellated the brain of each subject into 1,000 cortical regions of interest (ROIs; Figure 1C). We opted for this atlas 
as it provides a fine-grained segmentation of the cerebral cortex that, at the same time, reflects the seven resting state networks identified by Yeo and collaborators (2011): visual, somato-motor, ventral attention, dorsal attention, limbic, control and default mode network. To compute static ISFC, the time course of brain activity was extracted from each ROI of one subject and correlated using Pearson's product-moment coefficient with the remaining 999 regions of all other subjects. By repeating this procedure for each of the 14 subjects, we generated 91 (i.e., number of all possible combinations of subjects) correlation matrices having 499,500 cells each (i.e., number of all possible combinations of ROIs). We then applied Fisher z-transformation and averaged correlation values across subjects to produce a group-level ISFC map.

To compute tISFC, one has first to determine the width of the moving-window (i.e., how many timepoints in a single window) and the degree of overlap between adjacent windows (i.e., how many timepoints are shared by two subsequent windows). Both parameters are often chosen arbitrarily, even though they may significantly affect the results. To limit the arbitrariness of this choice, we estimated the optimal window width as a function of the intersubject correlation in affective dimensions (obtained from the behavioral experiment). Specifically, we computed the average intersubject correlation of affective dimension timeseries for all the possible window widths, ranging from 20 to 1000 timepoints (i.e., from 40s to $33 \mathrm{~m}$ ) and having 33\% of overlap (Figure 1A and 1B). We considered the optimal window size the point at which the intersubject correlation curve starts to flatten (i.e., knee point), namely the point representing a reasonable trade-off between the agreement across participants and the window width (Figure 1B). This procedure was repeated for all the affective dimensions separately. Once the optimal window width was established, we computed the tISFC as the Pearson's correlation between each ROI of one subject and all the other ROIs of the other subjects, across all the windows (Figure 1C). By repeating this procedure for each of the 14 subjects, we generated for each window 91 correlation matrices having 499,500 cells each (i.e., number of all possible combinations of ROIs). We then applied Fisher z-transformation and averaged 
correlation values across subjects to produce a group-level timeseries of brain connectivity (i.e., tISFC) associated with movie watching.

Experiment 1: fMRI Study - Association between tISFC and Changes in Affective Dimensions. The primary aim of our study was to relate changes in tISFC to variations in the perceived affective state during movie watching. Therefore, for each ROI we computed connectivity strength as the sum of the Z-transformed correlation values at each timepoint (Figure 1D). We thus obtained 1,000 timeseries expressing the relationship of each ROI with the rest of the brain throughout the movie. These timeseries were then correlated using Spearman's $\rho$ coefficient with the group-level timecourse of affective dimensions, after those were downsampled to the temporal resolution determined by the optimal window (see above) using a moving-average procedure. To assess the significance of the association (Figure 1E), we used a non-parametric permutation test based on timepoint shuffling of affective dimensions (200,000 iterations; minimum two-tailed p-value: 1.0e-5). At each iteration, the randomized affective dimensions were correlated with each of the 1,000 timeseries of ROI connectivity strength, providing null distributions of Spearman's correlation values. The position of the actual association in the null distribution determined the two-tails level of significance, which was then corrected for multiple comparisons using the Bonferroni method ( $n=1,000$; critical $p$-value was 5.0e-5).

Experiment 1: fMRI Study - Network Reconfigurations Related to Affective Dimensions. The parcellation proposed by Schaefer and colleagues (2018) is based on the seven resting state networks identified by Yeo and collaborators (2011). This allowed us to better characterize the relationship between changes in affective dimensions and functional characteristics of brain networks. Specifically, node strength summarizes the connectivity of a specific region with the rest of the brain, yet it does not provide any information about how brain networks reorganize as a function of the affective experience and which associations between ROIs are changing to a greater extent. To 
overcome this limitation, we first used classical Multi-Dimensional Scaling (MDS; Torgerson, 1952) on the group-level ISFC to measure and graphically represent functional distances of the 1,000 brain regions during movie watching. In the MDS plot, each region was color-coded in accordance with network membership and scaled in size and transparency depending on the log p-value of the association between connectivity strength and changes in affective dimensions. Results of this procedure are represented in Figure 2B and Figure 4B.

Moreover, we considered as a seed region each ROI found to be significant in previous analyses (see the Association between Time-Varying ISFC and Changes in Affective Dimensions paragraph) and represented its correlation with all other ROIs at tISFC timepoints corresponding to affective dimension scores either greater than the $90^{\text {th }}$ percentile or smaller than the $10^{\text {th }}$ percentile. Lastly, to better highlight how brain networks did reorganize at timepoints having opposite affective dimension scores, for each target region we computed the difference of correlation values and scaled its size and transparency accordingly. Results of this procedure are represented in Figure 3, Figure 5 and Supplementary Figure 2.

Experiment 1: fMRI Study - Timescales of the Relationship between Connectivity Strength and Affective Dimensions. Recent studies on narrative comprehension and memory encoding demonstrated that distinct brain regions segment and process information at specific timescales, with early and mid sensory areas preferring short events, and fronto-parietal association regions encoding hundreds of seconds of information (Baldassano et al., 2017). Starting from this evidence, we measured the preferred timescale at which brain regions process affective information. First, we repeated the estimate of the optimal window width (see Time-varying Intersubject Functional Correlation paragraph) at three additional timescales: from 20 to 250 , to 500 and to 750 timepoints. After the optimal width was determined for each range, we calculated the tISFC and correlated the connectivity strength of each ROI with the downsampled version of the affective dimensions. We then estimated the significance of the associations at additional timescales following the same 
procedure described above and applied a winner-takes-it-all criterion on the log-transformed p-value maps to establish the preferred timescale of each brain region. Results of this procedure are presented in Figure 2D and Figure 4D.

Experiment 2: Behavioral Study - Participants. Twenty-one Italian native speakers took part in the study (13F; mean age 29.4 years, range 23-44) and provided ratings of their moment-by-moment emotional experience while watching an edited version of the live action movie 101 Dalmatians (S. Herek, Walt Disney Pictures, 1996). All participants were clinically healthy and had no history of any neurological or psychiatric condition. Each subject was informed about the nature of the research and gave written informed consent to participate in the study. The local Ethical Review Board approved the experimental protocol and procedures (CEAVNO: Comitato Etico Area Vasta Nord Ovest; Protocol No. 1485/2017) and the research was conducted in accordance with the Declaration of Helsinki.

Experiment 2: Behavioral Study - Stimuli and Experimental Paradigm. A shortened version of 101 Dalmatians was selected as a validation dataset. Similarly to what Hanke and colleagues (2016) did in Forrest Gump (i.e., exploratory dataset), we discarded scenes irrelevant to the central plot, so as to maintain the total running time of the movie below 1 hour (i.e., 54 minutes). Video editing was carried out using the iMovie software (10.1.10) on an Apple Macbook Air. The movie was also split into six runs of similar duration and a six-second fade-in and fade-out period was added at the beginning and the end of each run.

In the behavioral experiment subjects sat comfortably in a silent room facing a 24" Dell ${ }^{\mathrm{TM}}$ screen, wore headphones (Marshall ${ }^{\mathrm{TM}}$ Major III; 20-20,000 Hz; Maximum SPL $97 \mathrm{~dB}$ ), and were asked to report their affective experience while watching 101 Dalmatians. As for the exploratory dataset, all participants have not watched the movie in the period of at least one year before the experiment. 
Stimulus presentation and recording of the responses were implemented in Matlab (R2019b; MathWorks Inc., Natick, MA, USA) and Psychtoolbox v3.0.16 (Kleiner et al., 2007).

Experiment 2: Behavioral Study - Single-Subject Affective Dimensions. For the exploratory dataset, we collected the perceived intensity of six emotions and then used PCA to obtain timeseries of polarity and intensity. In the validation dataset, instead, we modified the collection of behavioral reports, focusing directly on affective dimensions. In fact, participants were asked to report momentby-moment (i.e., $10 \mathrm{~Hz}$ sampling rate; 16,140 timepoints) the pleasantness or unpleasantness and intensity of the experience on a continuous scale ranging from -100 (extremely negative) to +100 (extremely positive). For each subject, raw ratings were interpreted as a measure of polarity, whereas its absolute value (i.e., the emotional impact regardless of the valence of the experience) was considered a measure of intensity. Lastly, to assess the agreement across subjects of polarity and intensity, we computed pairwise Spearman's correlation and its significance.

Experiment 2: fMRI Study - Participants. Brain activity elicited by the watching of 101 Dalmatians was obtained from ten healthy Italian subjects ( $8 \mathrm{~F} ; 35 \pm 13$ years), instructed to simply inhibit any movement and enjoy the movie. The fMRI acquisition lasted 1 hour and was split in six runs (3T Philips Ingenia scanner, Neuroimaging centre of NIT - Molinette Hospital, Turin; 32 channels head coil; gradient recall echo-echo planar imaging; $2000 \mathrm{~ms}$ repetition time, $30 \mathrm{~ms}$ echo time, $75^{\circ}$ flip angle, $3 \mathrm{~mm}$ isotropic voxel, $240 \mathrm{~mm}$ field of view). Audio and visual stimulation were delivered through MR-compatible LCD goggles and headphones (VisualStim Resonance Technology, video resolution $800 \times 600$ at $60 \mathrm{~Hz}$, visual field $30^{\circ} \times 22^{\circ}, 5^{\prime \prime}$, audio $30 \mathrm{~dB}$ noise-attenuation, $40 \mathrm{~Hz}$ to 40 $\mathrm{kHz}$ frequency response). Together with the fMRI data, 3D T1w high-resolution anatomical images were also acquired (magnetization-prepared rapid gradient echo; $7 \mathrm{~ms}$ repetition time, $3.2 \mathrm{~ms}$ echo time, $9^{\circ}$ flip angle, $1 \mathrm{~mm}$ isotropic voxel, $224 \mathrm{~mm}$ field of view). The fMRI study was approved by 
the Ethical Committee of the University of Turin (Protocol No. 195874/2019) and participants gave their written consent for the participation.

Experiment 2: fMRI Study - Data Analysis. Considering the high variability in results related to analytical choices (Botvinik-Nezer et al., 2020), here we tested two alternative pre-processing pipelines (i.e., workflow A and B) for the analysis of the validation dataset.

Specifically, for workflow A, we removed scanner-related noise by applying a spike removal procedure (3dDespike). Afterwards, all volumes were temporally aligned (3dTshift) and corrected for head motion ( $3 d$ volreg) by computing the displacement between each volume and the most stable timepoint of the first run (i.e., the one showing smallest framewise displacement values; Power et al., 2012). We applied a spatial smoothing up to 6mm (Gaussian kernel; $3 d B l u r T o F W H M$ ) and data were normalized (i.e., mean centering and percentage scaling). Moreover, we removed the trend in data by applying the Savitzky-Golay filtering (MATLAB function sgolayfilt, polynomial order: 3, frame length: 200 timepoints; Çukur et al., 2013). Runs were then concatenated, and multiple regression analysis was performed ( 3 dDeconvolve) to remove artifacts in signal related to head motion (6 motion parameters and spike regression for timepoint characterized by framewise displacement above 0.3 ). The residuals of this fitting were considered the single-subject brain activity associated with the watching of 101 Dalmatians, they were nonlinearly (3dQWarp) registered to the MNI standard space (Fonov et al., 2009) and used in subsequent analyses.

As a further control of the robustness of our findings, in workflow B, we followed the exact same pre-processing pipeline used for the exploratory dataset (i.e., experiment 1).

Experiment 2: fMRI Study - Association between tISFC and Changes in Affective Dimensions. To evaluate the reliability and generalizability of the association between tISFC and the timecourse of affective dimensions reported in experiment 1, we tested whether brain connectivity dynamics explain changes in polarity and intensity in 101 Dalmatians. To this aim, we first obtained timeseries 
of affective dimensions from each subject and estimated the optimal window width based on the intersubject correlation in polarity and intensity ratings (from 20 to 112 timepoints, $33 \%$ of overlap). Similarly to experiment 1 , we considered the point at which the intersubject correlation curve starts to flatten (i.e., knee point) as the optimal window size. Afterwards, we estimated the tISFC as the Pearson's correlation between each ROI of one subject and all the other ROIs of the other subjects, across all the windows. We then applied Fisher z-transformation and averaged correlation values across pairings of subjects to produce a group-level timeseries of brain connectivity related to the watching of 101 Dalmatians. Also, by summing the Z-transformed correlation values at each timepoint, we obtained a timeseries of connectivity strength for each ROI. As in experiment 1, grouplevel polarity and intensity timeseries were downsampled to the optimal window width using a moving-average procedure and they were correlated to connectivity strength of each ROI over time (Spearman's $\rho$ coefficient). To assess the significance of the association, we used a non-parametric permutation test based on timepoint shuffling of affective dimensions (200,000 iterations; minimum two-tailed p-value: 1.0e-5). Importantly, we restricted analyses to ROIs significantly tracking changes in polarity and intensity in experiment 1 , considering all explored timescales (i.e., logical OR between maps of polarity at 89, 143, 239 and 273 timepoints; logical OR between maps of intensity at 100, 163, 247 and 331 timepoints). Lastly, to control for false positives in experiment 2, we adjusted the significance threshold based on the number of tested ROIs ( $p<0.05$ Bonferroni corrected). This entire procedure was repeated for each analysis workflow (see previous section). 


\section{Acknowledgements}

We would like to thank all the people behind the studyforrest project, especially Michael Hanke. G.L., G.H. and L.C. are supported by Progetto di Attività Integrate - PAI Project - granted by IMT School for Advanced Studies Lucca. M.D. is supported by an ERC Consolidator Grant 2017 (LIGHTUP, project \# 772953).

\section{Author Contributions}

G.L., G.H., P.P. and L.C., conceived the study. G.L., G.H. and L.C. designed the behavioral experiment, collected behavioral data, developed the code, performed behavioral and fMRI data analysis, interpreted the obtained results and drafted the manuscript. E.M.C. acquired behavioral data of the validation dataset. F.S., V.B., M.D. and A.L. acquired fMRI data of the validation dataset. G.L., G.H., L.C., F.S. and A.L. analyzed fMRI data of the validation dataset. G.L., G.H., E.M.C., F.S., V.B., M.D., A.L., E.R., P.P. and L.C. critically revised the manuscript. All the authors approved the final version of the manuscript. 


\section{References}

1. Antony, J. W., Hartshorne, T. H., Pomeroy, K., Gureckis, T. M., Hasson, U., McDougle, S. D., \& Norman, K. A. (2021). Behavioral, physiological, and neural signatures of surprise during naturalistic sports viewing. Neuron, 109(2), 377-390.

2. Avants, B. B., Tustison, N., \& Song, G. (2009). Advanced normalization tools (ANTS). Insight j, 2(365), 1-35.

3. Baldassano, C., Chen, J., Zadbood, A., Pillow, J. W., Hasson, U., \& Norman, K. A. (2017). Discovering event structure in continuous narrative perception and memory. Neuron, 95(3), 709721.

4. Baucom, L. B., Wedell, D. H., Wang, J., Blitzer, D. N., \& Shinkareva, S. V. (2012). Decoding the neural representation of affective states. Neuroimage, 59(1), 718-727.

5. Botvinik-Nezer, R., Holzmeister, F., Camerer, C. F., Dreber, A., Huber, J., Johannesson, M., ... \& Rieck, J. R. (2020). Variability in the analysis of a single neuroimaging dataset by many teams. Nature, 582(7810), 84-88.

6. Bzdok, D., Schilbach, L., Vogeley, K., Schneider, K., Laird, A. R., Langner, R., \& Eickhoff, S. B. (2012). Parsing the neural correlates of moral cognition: ALE meta-analysis on morality, theory of mind, and empathy. Brain Struct Funct, 217(4), 783-796.

7. Chen, J., Leong, Y. C., Honey, C. J., Yong, C. H., Norman, K. A., \& Hasson, U. (2017). Shared memories reveal shared structure in neural activity across individuals. Nat Neurosci, 20(1), 115125.

8. Ciric, R., Wolf, D. H., Power, J. D., Roalf, D. R., Baum, G. L., Ruparel, K., ... \& Gur, R. C. (2017). Benchmarking of participant-level confound regression strategies for the control of motion artifact in studies of functional connectivity. Neuroimage, 154, 174-187.

9. Costa, T., Cauda, F., Crini, M., Tatu, M. K., Celeghin, A., de Gelder, B., \& Tamietto, M. (2014). Temporal and spatial neural dynamics in the perception of basic emotions from complex scenes. Soc Cogn Affect Neurosci, 9(11), 1690-1703.

10. Cox, R. W. (1996). AFNI: software for analysis and visualization of functional magnetic resonance neuroimages. Comput Biomed Res, 29(3), 162-173.

11. Çukur, T., Nishimoto, S., Huth, A. G., \& Gallant, J. L. (2013). Attention during natural vision warps semantic representation across the human brain. Nat Neurosci, 16(6), 763-770.

12. Ekman, P. (1992). An argument for basic emotions. Cogn Emot, 6(3-4), 169-200.

13. Etkin, A., Büchel, C., \& Gross, J. J. (2015). The neural bases of emotion regulation. Nat Rev Neurosci, 16(11), 693-700. 
14. Finn, E. S., Corlett, P. R., Chen, G., Bandettini, P. A., \& Constable, R. T. (2018). Trait paranoia shapes inter-subject synchrony in brain activity during an ambiguous social narrative. Nat Comm, 9(1), 1-13.

15. Fonov, V. S., Evans, A. C., McKinstry, R. C., Almli, C. R., \& Collins, D. L. (2009). Unbiased nonlinear average age-appropriate brain templates from birth to adulthood. Neuroimage, (47), S102.

16. Gao, W., \& Lin, W. (2012). Frontal parietal control network regulates the anti- correlated default and dorsal attention networks. Hum Brain Mapp, 33(1), 192-202.

17. Garvert, M. M., Friston, K. J., Dolan, R. J., \& Garrido, M. I. (2014). Subcortical amygdala pathways enable rapid face processing. Neuroimage, 102, 309-316.

18. Hanke, M., Adelhöfer, N., Kottke, D., Iacovella, V., Sengupta, A., Kaule, F. R., ... \& Stadler, J. (2016). A studyforrest extension, simultaneous fMRI and eye gaze recordings during prolonged natural stimulation. Sci Data, 3, 160092.

19. Harvey, B. M., Klein, B. P., Petridou, N., \& Dumoulin, S. O. (2013). Topographic representation of numerosity in the human parietal cortex. Science, 341(6150), 1123-1126.

20. Hasson, U., Nir, Y., Levy, I., Fuhrmann, G., \& Malach, R. (2004). Intersubject synchronization of cortical activity during natural vision. Science, 303(5664), 1634-1640.

21. Kim, J., Shinkareva, S. V., \& Wedell, D. H. (2017). Representations of modality-general valence for videos and music derived from fMRI data. Neuroimage, 148, 42-54.

22. Kleckner, I. R., Zhang, J., Touroutoglou, A., Chanes, L., Xia, C., Simmons, W. K., ... \& Barrett, L. F. (2017). Evidence for a large-scale brain system supporting allostasis and interoception in humans. Nat Hum Behav, 1(5), 1-14.

23. Kleiner, M. et al. What's new in Psychtoolbox-3. Perception 36, 1 (2007).

24. Kohn, N., Eickhoff, S. B., Scheller, M., Laird, A. R., Fox, P. T., \& Habel, U. (2014). Neural network of cognitive emotion regulation - an ALE meta-analysis and MACM analysis. Neuroimage, 87, 345-355.

25. Kragel, P. A., Knodt, A. R., Hariri, A. R., \& LaBar, K. S. (2016). Decoding spontaneous emotional states in the human brain. PLoS Biol, 14(9), e2000106.

26. Kuppens, P., Oravecz, Z., \& Tuerlinckx, F. (2010). Feelings change: accounting for individual differences in the temporal dynamics of affect. J Pers Soc Psychol, 99(6), 1042.

27. Kuppens, P., \& Verduyn, P. (2017). Emotion dynamics. Curr Opin Psychol, 17, 22-26.

28. Lamm, C., Nusbaum, H. C., Meltzoff, A. N., \& Decety, J. (2007). What are you feeling? Using functional magnetic resonance imaging to assess the modulation of sensory and affective responses during empathy for pain. PloS One, 2(12). 
29. Lettieri, G., Handjaras, G., Ricciardi, E., Leo, A., Papale, P., Betta, M., ... \& Cecchetti, L. (2019). Emotionotopy in the human right temporo-parietal cortex. Nat Comm, 10(1), 1-13.

30. Lindquist, K. A., \& Barrett, L. F. (2012). A functional architecture of the human brain: emerging insights from the science of emotion. Trends Cogn Sci, 16(11), 533-540.

31. Margulies, D. S., Ghosh, S. S., Goulas, A., Falkiewicz, M., Huntenburg, J. M., Langs, G., ... \& Jefferies, E. (2016). Situating the default-mode network along a principal gradient of macroscale cortical organization. PNAS, 113(44), 12574-12579.

32. Mirchi, N., Betzel, R. F., Bernhardt, B. C., Dagher, A., \& Mišić, B. (2019). Tracking mood fluctuations with functional network patterns. Soc Cogn Affect Neurosci, 14(1), 47-57.

33. Nastase, S. A., Gazzola, V., Hasson, U., \& Keysers, C. (2019). Measuring shared responses across subjects using intersubject correlation. BioRxiv, 600114.

34. Pessoa, L. (2017). A network model of the emotional brain. Trends Cogn Sci, 21(5), 357-371.

35. Pessoa, L. (2018). Understanding emotion with brain networks. Curr Opin Behav Sci, 19, 19-25.

36. Posner, J., Russell, J. A., Gerber, A., Gorman, D., Colibazzi, T., Yu, S., ... \& Peterson, B. S. (2009). The neurophysiological bases of emotion: An fMRI study of the affective circumplex using emotion- denoting words. Hum Brain Mapp, 30(3), 883-895.

37. Power, J. D., Barnes, K. A., Snyder, A. Z., Schlaggar, B. L., \& Petersen, S. E. (2012). Spurious but systematic correlations in functional connectivity MRI networks arise from subject motion. Neuroimage, 59(3), 2142-2154.

38. Protopapa, F., Hayashi, M. J., Kulashekhar, S., van der Zwaag, W., Battistella, G., Murray, M. M., ... \& Bueti, D. (2019). Chronotopic maps in human supplementary motor area. PLoS Biol, $17(3)$.

39. Raichle, M. E. (2015). The brain's default mode network. Annu Rev Neurosci, 38, 433-447.

40. Raz, G., Touroutoglou, A., Wilson-Mendenhall, C., Gilam, G., Lin, T., Gonen, T., ... \& MaronKatz, A. (2016). Functional connectivity dynamics during film viewing reveal common networks for different emotional experiences. Cogn Affect Behav Neurosci, 16(4), 709-723.

41. Résibois, M., Rotgé, J. Y., Delaveau, P., Kuppens, P., Van Mechelen, I., Fossati, P., \& Verduyn, P. (2018). The impact of self-distancing on emotion explosiveness and accumulation: An fMRI study. PloS One, 13(11), e0206889.

42. Résibois, M., Verduyn, P., Delaveau, P., Rotgé, J. Y., Kuppens, P., Van Mechelen, I., \& Fossati, P. (2017). The neural basis of emotions varies over time: different regions go with onset-and offset-bound processes underlying emotion intensity. Soc Cogn Affect Neurosci, 12(8), 12611271. 
43. Satpute, A. B., \& Lindquist, K. A. (2019). The Default Mode Network's Role in Discrete Emotion. Trends Cogn Sci.

44. Saxe, R., \& Kanwisher, N. (2003). People thinking about thinking people: the role of the temporo-parietal junction in "theory of mind". Neuroimage, 19(4), 1835-1842.

45. Schaefer, A., Kong, R., Gordon, E. M., Laumann, T. O., Zuo, X. N., Holmes, A. J., ... \& Yeo, B. T. (2018). Local-global parcellation of the human cerebral cortex from intrinsic functional connectivity MRI. Cereb Cortex, 28(9), 3095-3114.

46. Schaefer, A., Nils, F., Sanchez, X., \& Philippot, P. (2010). Assessing the effectiveness of a large database of emotion-eliciting films: A new tool for emotion researchers. Cogn Emot, 24(7), 1153-1172.

47. Schilbach, L., Eickhoff, S. B., Rotarska-Jagiela, A., Fink, G. R., \& Vogeley, K. (2008). Minds at rest? Social cognition as the default mode of cognizing and its putative relationship to the "default system" of the brain. Conscious Cogn, 17(2), 457-467.

48. Shamay-Tsoory, S. G., Tomer, R., Berger, B. D., Goldsher, D., \& Aharon-Peretz, J. (2005). Impaired "affective theory of mind" is associated with right ventromedial prefrontal damage. Cogn Behav Neurol, 18(1), 55-67.

49. Simony, E., Honey, C. J., Chen, J., Lositsky, O., Yeshurun, Y., Wiesel, A., \& Hasson, U. (2016). Dynamic reconfiguration of the default mode network during narrative comprehension. Nat Comm, 7, 12141.

50. Thornton, M. A., \& Tamir, D. I. (2017). Mental models accurately predict emotion transitions. PNAS, 114(23), 5982-5987.

51. Torgerson, W. S. (1952). Multidimensional scaling: I. Theory and method. Psychometrika, 17(4), 401-419.

52. Trampe, D., Quoidbach, J., \& Taquet, M. (2015). Emotions in everyday life. PloS one, 10(12), $\mathrm{e} 0145450$.

53. Verduyn, P., Delaveau, P., Rotgé, J. Y., Fossati, P., \& Van Mechelen, I. (2015). Determinants of emotion duration and underlying psychological and neural mechanisms. Emot Rev, 7(4), 330335.

54. Verduyn, P., \& Lavrijsen, S. (2015). Which emotions last longest and why: The role of event importance and rumination. Mot Emot, 39(1), 119-127.

55. Waugh, C. E., \& Schirillo, J. A. (2012). Timing: a missing key ingredient in typical fMRI studies of emotion. Behav Brain Sci, 35(3), 170-171.

56. Waugh, C. E., Shing, E. Z., \& Avery, B. M. (2015). Temporal dynamics of emotional processing in the brain. Emot Rev, 7(4), 323-329. 
57. Yeo, B. T., Krienen, F. M., Sepulcre, J., Sabuncu, M. R., Lashkari, D., Hollinshead, M., ... \& Fischl, B. (2011). The organization of the human cerebral cortex estimated by intrinsic functional connectivity. J Neurophysiol, 106(3), 1125-1165.

58. Yeshurun, Y., Nguyen, M., \& Hasson, U. (2021). The default mode network: where the idiosyncratic self meets the shared social world. Nat Rev Neurosci, 1-12. 